\title{
When and why to start continuous renal replacement therapy in critically ill patients with acute kidney injury
}

\author{
Jung Nam $\mathrm{An}^{1}$, Sung Gyun $\mathrm{Kim}^{1,2}$, Young Rim Song ${ }^{1,2,3}$ \\ ${ }^{1}$ Division of Nephrology, Department of Internal Medicine, Hallym University Sacred Heart Hospital, Anyang, Republic of Korea \\ ${ }^{2}$ Hallym University Kidney Research Institute, Anyang, Republic of Korea \\ ${ }^{3}$ Department of Biomedical Gerontology, Graduate School of Hallym University, Chuncheon, Korea
}

\begin{abstract}
Acute kidney injury (AKI) is a common condition in critically ill patients, and may contribute to significant medical, social, and economic consequences, including death. Although there have been advances in medical technology, including continuous renal replacement therapy (CRRT), the mortality rate of AKI is high, and there is no fundamental treatment that can reverse disease progression. The decision to implement CRRT is often subjective and based primarily on the clinician's judgment without consistent and concrete guidelines or protocols regarding when to initiate and discontinue CRRT and how to manage complications. Recently, several randomized controlled trials addressing the initiation of renal replacement therapy in critically ill patients with AKI have been completed, but clinical application of the findings is limited by the heterogeneity of the objectives and research designs. In this review, the advantages and disadvantages of CRRT initiation, clinical guideline recommendations, and the results of currently published clinical trials and meta-analyses are summarized to guide patient care and identify future research priorities.
\end{abstract}

Keywords: Acute kidney injury, Continuous renal replacement therapy, Guideline, Meta-analysis, Randomized controlled trial

\section{Introduction}

Acute kidney injury (AKI) is a common complication in critically ill patients [1,2], and it increases the risk of morbidity and mortality, including progression to chronic kidney disease [3,4], major adverse cardiac events [5-7], infection $[8,9]$, and gastrointestinal bleeding [10]. The number of AKI cases requiring renal replacement therapy (RRT) in intensive care units (ICUs) continues to increase worldwide [11-14]. Despite remarkable medical advances, including the development of continuous RRT (CRRT), the mortality rate of patients with severe AKI requiring RRT is as high as $60 \%$ to $70 \%$ [15-18]. No treatment can completely resolve the course of AKI, and conservative and supportive treatments remain the mainstay of clinical management. Supportive CRRT is an important tool to improve the prognosis of patients with severe AKI [19-21]; however, there are no clear guidelines for its use or concrete evidence supporting the various clinical protocols. Despite the absence of clear criteria or guidelines for selection of CRRT rather than in-

Received: February 17, 2021; Revised: July 9, 2021; Accepted: July 22, 2021

Editor: Kent Doi, The University of Tokyo Hospital, Tokyo, Japan

Correspondence: Young Rim Song

Division of Nephrology, Department of Internal Medicine, Hallym University Sacred Heart Hospital, 22 Gwanpyeong-ro 170beon-gil, Dongan-gu, Anyang 14068, Republic of Korea. E-mail: youngrim@hallym.or.kr

ORCID: https://orcid.org/0000-0002-0416-4745

Copyright (@ 2021 by The Korean Society of Nephrology

(a) This is an Open Access article distributed under the terms of the Creative Commons Attribution Non-Commercial and No Derivatives License (http:// creativecommons.org/licenses/by-nc-nd/4.0/) which permits unrestricted non-commercial use, distribution of the material without any modifications, and reproduction in any medium, provided the original works properly cited. 
termittent hemodialysis as the RRT modality, use of CRRT is recommended in cases of hemodynamic instability even when the criteria are appropriate for intermittent hemodialysis [2]. The question of when to initiate CRRT has been discussed extensively in leading journals in the field of intensive care medicine and nephrology in recent years, but it remains controversial.

When should CRRT be initiated? Specifically, initiation of CRRT as an emergency intervention is indicated in cases of life-threatening, medically refractory complications of AKI (Table 1) [22]. This is a situation where the patient will die if CRRT is not started immediately. However, in clinical practice, this situation does not occur often, and there are not many cases where CRRT is implemented based only on this indication. Unless there is an obvious emergent indication, it is important for the clinician to use their judgment and consider the severity of the accompanying clinical situation, dysfunction of other organs (brain, heart, lung, liver, and gastrointestinal tract), and possibility of renal function recovery. In addition, the preferred treatment option selected by the patient, caregiver, and physician must be considered, as well as the cost of treatment and circumstances of each institution, including its internal protocols/guidelines and available infrastructure (resources). The basic premise is that the benefits of implementing CRRT should outweigh the associated risks [23].
This concise review summarizes the results of research addressing implementation of CRRT and compiles current guidelines regarding indications for and initiation of CRRT in critically ill patients with AKI. The review focuses on the findings of the latest randomized controlled trials (RCTs). The aim is to provide relevant insights to supplement the clinician's judgment.

\section{Advantages of early continuous renal replacement therapy}

The advantages of early CRRT implementation in the absence of traditional indications include avoidance and/or early control of fluid accumulation and overload, acid-base and electrolyte/metabolic derangement, complications of uremia, and unnecessary or excessive diuretic exposure. Early CRRT also can support beneficial immunomodulation and increase clearance of inflammatory mediators (Table 2) [22]. Thus, early CRRT is necessary to maintain fluid, electrolyte, and acid-base homeostasis, as well as to treat and prevent life-threatening AKI-related complications and deterioration of organs other than the kidneys. Notably, early CRRT becomes necessary when there is hemodynamic instability, fluid overload, catabolism, and/or sepsis with severe AKI to the extent that it is difficult to apply intermittent hemodialysis [2]. These criteria are different from those

Table 1. Indications and contraindications for CRRT initiation in critically ill patients with AKI

\begin{tabular}{|c|c|}
\hline \multirow{5}{*}{$\begin{array}{l}\text { Absolute indications (in the absence of } \\
\text { contraindications for CRRT) }\end{array}$} & Refractory hyperkalemia \\
\hline & Refractory metabolic acidosis \\
\hline & Refractory pulmonary edema due to volume overload not responding to diuretics \\
\hline & Symptomatic uremia or its complications (bleeding, pericarditis, encephalopathy, etc.) \\
\hline & Overdose or toxicity of dialyzable drugs (salicylates, ethylene glycol, methanol, etc.) \\
\hline \multirow{4}{*}{$\begin{array}{l}\text { Relative indications (in the absence of } \\
\text { life-threatening complications of AKI) }\end{array}$} & Hemodynamic instability \\
\hline & $\begin{array}{l}\text { Advanced dysfunction of organs other than the kidneys (brain, heart, lung, liver, and gastrointesti- } \\
\text { nal tract) }\end{array}$ \\
\hline & $\begin{array}{l}\text { Need for administration of a large volume of fluid (massive volume challenge, massive transfu- } \\
\text { sion, medications, nutritional support, etc.) }\end{array}$ \\
\hline & Severity of the underlying disease \\
\hline \multirow[t]{2}{*}{ Contraindications } & Patient or legal representative does not want CRRT \\
\hline & No infrastructure or skilled manpower to administer CRRT \\
\hline \multirow[t]{2}{*}{ Relative contraindications } & Futile prognosis \\
\hline & Patient receiving palliative care \\
\hline
\end{tabular}

AKI, acute kidney injury; CRRT, continuous renal replacement therapy.

Reproduced from the article of Ostermann et al. (Contrib Nephrol 2016;187:106-120) [22] with the permission from S. Karger AG. 
Table 2. Advantages and disadvantages of early continuous renal replacement therapy in critically ill patients with acute kidney injury

\begin{tabular}{|c|c|}
\hline Advantage & Disadvantage \\
\hline $\begin{array}{l}\text { Avoidance and/or earlier control of fluid accumulation and volume } \\
\text { overload }\end{array}$ & $\begin{array}{l}\text { Risk of iatrogenic episodes of hemodynamic instability that can wors- } \\
\text { en the clinical situation or delay patient recovery }\end{array}$ \\
\hline Avoidance and/or earlier control of complications of uremia & Need for and complications associated with anticoagulation therapy \\
\hline $\begin{array}{l}\text { Avoidance and/or earlier control of acid-base/metabolic disorders } \\
\text { and electrolyte abnormalities }\end{array}$ & $\begin{array}{l}\text { Need for and complications associated with dialysis catheterization } \\
\text { (bleeding, bloodstream infection, etc.) }\end{array}$ \\
\hline Avoidance of unnecessary or excessive diuretics & Need for and complications associated with immobilization \\
\hline Stability of intracranial pressure & Risk of excess clearance of micronutrients and trace elements \\
\hline Immune regulation and active removal of inflammatory mediators & $\begin{array}{l}\text { Risk of excess clearance of vital medications (antibiotics, anticonvul- } \\
\text { sants, etc.) below therapeutic levels }\end{array}$ \\
\hline \multicolumn{2}{|c|}{$\begin{array}{l}\begin{array}{l}\text { Relief of further injuries or burden by taking the place of the damaged Unnecessary exposure to renal replacement therapy among patients } \\
\text { kidney function } \\
\text { who could recover kidney function spontaneously with only conser- } \\
\text { vative management }\end{array}\end{array}$} \\
\hline & $\begin{array}{l}\text { Increased costs of treatment, use of resources, and manpower } \\
\text { required. }\end{array}$ \\
\hline
\end{tabular}

Reproduced from the article of Ostermann et al. (Contrib Nephrol 2016;187:106-120) [22] with the permission from S. Karger AG.

applied when initiating intermittent dialysis in end-stage renal disease, and the indications can be diverse and broad depending on the clinician's judgment and criteria.

\section{Disadvantages of early continuous renal replacement therapy}

One significant limitation of early CRRT is the high risk of a variety of complications (Table 2) [22]. Complications such as bleeding, infection, and pneumothorax can be caused by placement of the central venous catheter, and anticoagulant treatment is required to maintain the circuit and filter. In addition, CRRT typically is initiated for patients who are hemodynamically unstable and unable to withstand intermittent dialysis. However, complications such as blood pressure decrease, tachycardia, and other arrhythmia can occur during CRRT and worsen the clinical situation or delay patient recovery. In addition, micronutrients, trace elements, and/or therapeutic drugs can be cleared more rapidly through the CRRT circuit, resulting in low or inconsistent concentrations in blood and tissues and potentially leading to adverse events and/or loss of therapeutic effect. The use of antibiotics or anticonvulsants can be problematic if blood concentrations cannot be monitored. In severe AKI requiring CRRT due to sepsis, the most important treatment objective is appropriate use of antibiotics. If the blood concentration of antibiotics is not maintained, it can affect patient prognosis.

Another potential issue with the use of CRRT is that ther- apy (and the accompanying risk of complications) can be initiated in patients whose renal function would have improved more rapidly had they received conservative management alone.

Finally, the use of CRRT creates hardship for the patient and requires significant use of hospital personnel and equipment. The patient must enter the ICU for CRRT and cannot move throughout the application of CRRT, one CRRT machine is required per patient, and real-time monitoring is needed. Additionally, it is necessary to have ICU staff available to change the CRRT fluid or respond to alarms, and resources such as filters, CRRT fluids, and catheters are consumed continuously. The biggest drawback is that the cost of providing CRRT significantly increases the cost of patient care. As such, it is necessary to make decisions that consider the potential benefits (whether survival or secondary outcomes) of early initiation of CRRT and the resulting economic or medical burden.

\section{Timing of continuous renal replacement therapy}

In cases where the advantages and disadvantages of CRRT are understood, the question is the need for CRRT without absolute indication. If the response is yes, when should CRRT be initiated?

As noted above, the potential benefits of early initiation of CRRT must be balanced with the risks and burdens associated with CRRT [24]. In clinical practice, the final outcome has been favorable in only a few clinical scenarios (Fig. 1) 


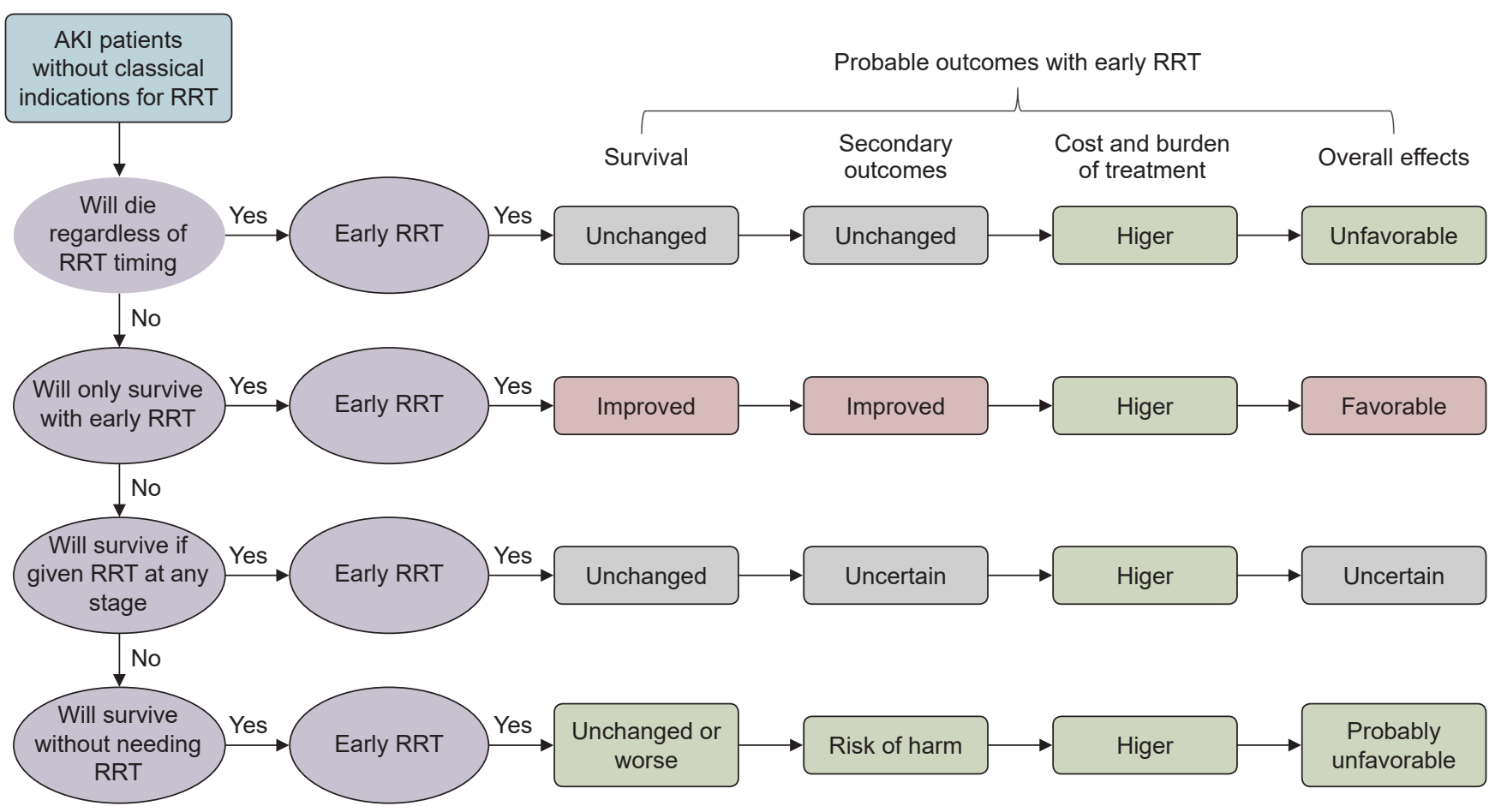

Figure 1. In the absence of absolute indications, predicting the prognosis of critically ill AKI patients undergoing early initiation of RRT is complex. Reproduced from the article of Prowle and Davenport (Kidney Int 2015;88:670-673) [25] with the permission from Elsevier.

AKI, acute kidney injury; RRT, renal replacement therapy.

[25]. Given RRT as an invasive treatment with significant physical, economic, medical, and social impacts, early initiation of RRT should be considered only after assessing the likelihood of patient survival and potential to achieve secondary outcomes, in addition to the survival of multiple organs, including the kidney. Considering the high cost and burden of treatment, the overall effect of RRT is favorable only in cases where RRT must be started early to ensure that the patient survives. In such cases, both survival and secondary outcomes are improved, resulting in an overall favorable effect. However, it is difficult to predict the optimal time of RRT initiation to achieve the greatest beneficial effect. Notably, early RRT should not be initiated for cases that are predicted to have unfavorable outcomes. For example, early RRT initiation can 'do harm' in situations where the patient is expected to die regardless of RRT or to survive without RRT. Therefore, predicting the final outcome of RRT initiation is a complex process that requires the consideration of different factors. It is necessary to understand and identify situations in which the patient will die if RRT is not started immediately and to make a quick decision.
Several observational studies and meta-analyses have reported that the early application of CRRT reduces mortality in critically ill patients with AKI [17,26-35]. However, the primary studies were observational, and the meta-analyses were based on observational studies; thus, their quality of evidence is low, and they have several limitations. First, only patients who started RRT were enrolled, and the prognosis was compared by classifying RRT as "early" or "late." Comparative analyses including patients who did not undergo RRT (i.e., patients whose renal function recovered or those who died without RRT) were not completed [36]. Second, the criteria or definitions for classifying "early" and "late" were arbitrary and differed by study. Third, the patients and their underlying diseases were heterogeneous. Fourth, various residual confounders could not be adjusted for, and some biases could not be controlled; hence, care should be taken when interpreting the results. Furthermore, more recent research results have been published indicating that early application of CRRT did not affect patient prognosis or recovery of renal function [37-41]. 


\section{Current recommendations for initiation of continuous renal replacement therapy}

The current clinical practice guidelines are summarized in Table 3. In 2012, the Kidney Disease: Improving Global Outcomes (KDIGO) study group [42] recommended emergency RRT in cases of potentially fatal changes in fluid, electrolytes, and/or acid-base balance. The trend of broader clinical situations and laboratory test results in addition to the values of serum creatinine or blood urea nitrogen should be examined, and it is recommended to assess and judge whether there are conditions to be modified through RRT. These recommendations were based on expert opinion, not evidence-based grading. In 2015, the French Intensive Care Society presented a similar expert opinion in which the results were deemed insufficient to define the appropriate timing for initiation of RRT beyond life-threatening indications [43]. Although the definitions of "early" and "late" initiation of CRRT were described, the quality of evidence was low. In the following year, the Japanese Clinical Practice Guideline was released, which also highlighted the lack of evidence to support decision making [44]. In 2019, the National Institute for Health and Care Excellence guidelines for AKI were revised, but the content was not significantly different from that released in 2013 [45]. To date, the published guidelines have emphasized the need for high-quality evidence from high-quality clinical trials to improve decision making.

\section{Recent studies addressing when to start continuous renal replacement therapy}

\section{Randomized controlled trials}

Well-designed RCTs published within the last 5 years have addressed the effect of the timing of RRT initiation on patient outcomes (Table 4). The Early Versus Late Initiation of RRT in Critically Ill Patients with Acute Kidney Injury (ELAIN) trial [46] was conducted in 231 patients. The early group had no conventional indications for CRRT, but the therapy was started within 8 hours of a diagnosis of KDIGO stage 2 AKI. The delayed group started CRRT within 12 hours of a diagnosis of KDIGO stage 3 AKI or when a conventional indication was present. Significant improvements in outcomes among the early CRRT group compared with the delayed group were reported with respect to 90 -day mortality (39.3\%

Table 3. Recommendations from clinical practice guidelines

\begin{tabular}{|c|c|}
\hline Guideline & Recommendation \\
\hline \multirow[t]{2}{*}{$\begin{array}{l}\text { Kidney Disease: Improving Global Outcomes } \\
\text { (KDIGO) [42] }\end{array}$} & $\begin{array}{l}\text { Initiate RRT emergently with life-threatening changes in fluid, electrolyte, and acid-base } \\
\text { balance. (Not graded) }\end{array}$ \\
\hline & $\begin{array}{l}\text { Consider the broader clinical context, the presence of conditions that can be modified by } \\
\text { RRT, and trends of laboratory tests (rather than BUN and creatinine thresholds alone) } \\
\text { when making the decision to start RRT. (Not graded) }\end{array}$ \\
\hline \multirow[t]{8}{*}{$\begin{array}{l}\text { National Institute for Health and Care Excel- } \\
\text { lence (NICE) [45] }\end{array}$} & $\begin{array}{l}\text { Discuss any potential indications for RRT with a nephrologist, pediatric nephrologist, and/or } \\
\text { critical care specialist to ensure that the therapy is started as soon as needed }\end{array}$ \\
\hline & $\begin{array}{l}\text { Refer adults, children, and young people immediately for RRT if any of the following are not } \\
\text { responding to medical management: }\end{array}$ \\
\hline & - Hyperkalemia \\
\hline & - Metabolic acidosis \\
\hline & - Symptoms or complications of uremia \\
\hline & - Fluid overload \\
\hline & - Pulmonary edema \\
\hline & $\begin{array}{l}\text { Base the decision to start RRT on the condition of the adult, child, or young person as a } \\
\text { whole and not on isolated urea, creatinine, or potassium value }\end{array}$ \\
\hline \multirow[t]{2}{*}{ French Intensive Care Society (SRLF) [43] } & $\begin{array}{l}\text { RRT should be initiated without delay in life-threatening situations, including hyperkalemia, } \\
\text { metabolic acidosis, and refractory pulmonary edema (Expert opinion; strong agreement) }\end{array}$ \\
\hline & $\begin{array}{l}\text { The available data are insufficient to define optimal timing of initiation of RRT outside of } \\
\text { life-threatening situations (Expert opinion; strong agreement) }\end{array}$ \\
\hline The Japanese Clinical Practice Guideline [44] & $\begin{array}{l}\text { There is little evidence to support the theory that early initiation of blood purification im- } \\
\text { proves the outcomes of AKI. Initiation of RRT should be based upon broad considerations } \\
\text { of the clinical symptoms and disease conditions (Not graded; C) }\end{array}$ \\
\hline
\end{tabular}

AKI, acute kidney injury; BUN, blood urea nitrogen; RRT, renal replacement therapy. 


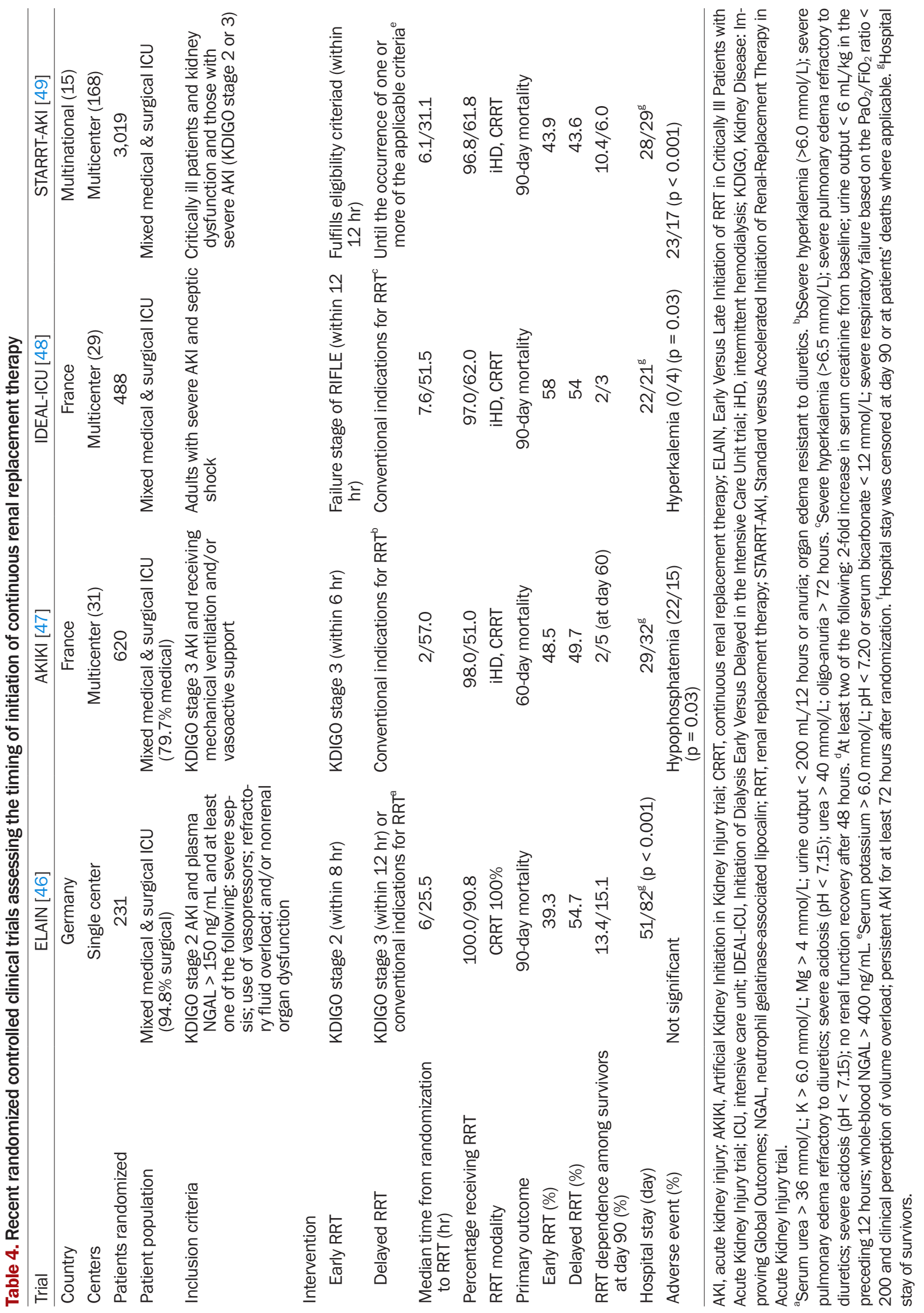


vs. $54.7 \%$; hazard ratio [HR], 0.66; 95\% confidence interval [CI], 0.45-0.97; $\mathrm{p}=0.03$ ), hospital stay (51 days vs. 82 days; HR, 0.34; 95\% CI, 0.22-0.52; $<<0.001$ ), and renal function recovery ( $53.6 \%$ vs. $38.7 \%$; odds ratio, 0.55 ; $95 \%$ CI, $0.32-0.93$; $\mathrm{p}=0.02$ ). However, excluding the patients who died within 3 months, the proportion of patients who recovered renal function at 90 days did not differ between the two groups. In this small RCT performed in a single-center, most patients underwent surgery, 91\% received RRT even though they were assigned to the delayed group, and the difference in time to initiate RRT between the groups was only 20 hours. Although the dialysis modality was unified as CRRT, it was difficult to view the study as well-controlled and designed. In addition, patients assigned to the early group might have recovered spontaneously without CRRT, leaving the possibility of skewed study results.

The Artificial Kidney Initiation in Kidney Injury (AKIKI) trial [47] of 620 patients with severe AKI admitted to 31 ICUs in France compared an early group that underwent CRRT within 6 hours of reaching KDIGO stage 3 AKI without conventional indication and a delayed group that underwent CRRT when a conventional indication was present. There were no differences in 60-day mortality between the early and delayed groups $(48.5 \%$ and $49.7 \%$, respectively; $\mathrm{p}=$ 0.79 ) or in the secondary outcomes, such as ventilator- and vasoactive-free days, ICU and hospital stays for 28 days, and dialysis dependence on day 60 . In the delayed group, $51 \%$ of patients received RRT compared with $98 \%$ in the early group ( $\mathrm{p}<0.001$ ). The number of RRT-free days was greater, and diuresis, an indicator of renal function improvement, appeared earlier in the delayed group than in the early group $(\mathrm{p}<0.001)$. However, with respect to the RRT modality used in the study, intermittent hemodialysis and CRRT were mixed, and there are limitations in generalization of the study findings.

Two years later, the Initiation of Dialysis Early Versus Delayed in the Intensive Care Unit (IDEAL-ICU) trial [48] reported the results of a study of 488 patients admitted into 29 ICUs in France for septic shock and associated severe AKI. The early group underwent RRT within 12 hours after meeting the Risk, Injury, Failure, Loss of kidney function, and End-stage kidney disease (RIFLE) criterion F without a conventional indication, and the delayed group underwent RRT if their condition did not improve within 48 hours after meeting the RIFLE criterion F or showed a conventional indication. The mortality rate at 90 days was not reduced in the early group compared to the delayed group (58\% and $54 \%$, respectively; $\mathrm{p}=0.38$ ). Ventilator-and vasoactive-free days and duration of stay in the ICU and hospital were also not different between the two groups, but the proportion of RRT recipients in the delayed group was smaller than that in the early group ( $97 \%$ vs. $62 \%$, respectively; $p<0.001)$. Given that the RIFLE criteria were not applied in earlier studies and the application of mixed intermittent dialysis and CRRT, direct comparison of the available study results is of limited utility.

The most recently completed Standard versus Accelerated Initiation of Renal-Replacement Therapy in Acute Kidney Injury (STARRT-AKI) trial [49] was a multinational, multicenter, open-label RCT that targeted 3,019 patients with severe AKI at 168 hospitals in 15 countries over a 4 -year period. There was no significant difference in mortality at 90 days between the group who started RRT within 12 hours after reaching KDIGO stage 2 or 3 AKI and the group that received RRT within 72 hours after randomization due to continued AKI or the presence of a conventional indication (early group vs. delayed group: $43.9 \%$ vs. $43.7 \%$; $\mathrm{p}=0.92$ ). However, among survivors at 90 days, the early group showed about 1.7-fold greater RRT dependence than the delayed group (risk ratio [RR], 1.7; 95\% CI, 1.2-2.4), and the percentage of patients who experienced adverse events during the RRT maintenance period was significantly greater in the early group than in the delayed group (23\% vs. $17 \%, \mathrm{p}<0.001$ ). Among the RCTs on related topics, the largest and relatively pragmatic design has been advocated, but the limitation is that individual clinicians' bias was involved in determining the eligibility of the study subjects. Furthermore, the median time to initiate RRT was 6.1 hours (interquartile range [IQR], 3.9-8.8 hours) from full eligibility judgment in the early group, whereas that in the delayed group, for which there was no obligation to select RRT and the judgment of individual clinicians was followed, was 31.1 hours (IQR, 19.0-71.8 hours).

It is difficult to make general recommendations to guide clinical practice due to the heterogeneity of the designs of the published RCTs. The four aforementioned RCTs (1) enrolled diverse study subjects (a mix of medical and surgical ICU patients); (2) applied different criteria for "early" and "delayed" CRRT initiation or were not consistent in their application (for example, the key entry criterion for both the 
AKIKI and IDEAL-ICU trial-stage 3 AKI-was the criterion for late initiation of RRT in the ELAIN trial); (3) the RRT modality was mixed; (4) patients with emergent indications, such as refractory hyperkalemia, metabolic acidosis, and pulmonary edema, were excluded from the AKIKI and IDEAL-ICU trials, whereas the majority of the patients in the ELAIN trial had fluid overload or pulmonary edema prior to enrollment, and the results cannot be said to reflect typical clinical practice; (5) most importantly, the criteria for initiating RRT used in the studies were contingent and specified for the study, and there was inconsistency with the situation of actual patients requiring RRT; and 6) the protocols for initiation or discontinuation of RRT differed by trial. Therefore, it is difficult to apply the research results to typical patient care and clinical situations.

In consideration of these points, RCTs should continue to be conducted and well-controlled with practical and applicable research designs. Furthermore, if a proven clinical or laboratory marker or tool that reliably can distinguish patients who are likely to require RRT from patients who can recover without RRT or predictive models using scoring or artificial intelligence are available, effective, and efficient treatment using limited resources will be possible, and unnecessary exposure to RRT will be minimized. Further research is expected in the future.

The results of the Artificial Kidney Initiation in Kidney Injury 2 (AKIKI2) trial that is currently in progress (ClinicalTrials.gov identifiers: AKIKI-2 [NCT03396757]) are expected to provide significant contributions to the body of knowledge regarding CRRT in AKI [50]. The AKIKI2 trial is a prospective, multicenter, open-label, two-arm randomized trial that comprises observational and randomization stages. Patients with KDIGO stage 3 AKI who need a vasopressor will be included in the observational stage (expected to be about 810 patients), and patients with serum urea concentration of $40 \mathrm{mmol} / \mathrm{L}$ or greater or oliguria/anuria for more than 72 hours will be randomized to receive RRT within 12 hours (the standard group). In contrast, in the delayed group, when the serum urea concentration reaches $50 \mathrm{mmol} / \mathrm{L}$ or more or a severe condition (e.g., severe hyperkalemia, severe metabolic or mixed acidosis, or acute pulmonary edema due to fluid overload resulting in severe hypoxemia) occurs, RRT will be initiated, and randomization of about 270 patients is expected. The primary outcome will be defined as the number of RRT-free days by day 28 .

\section{Meta-analyses}

The results of the available meta-analysis comparing the clinical effects of early or delayed initiation of RRT in critically ill patients with AKI are as follows.

In a meta-analysis of 1,636 patients from nine RCTs published from 1985 to 2016, early RRT initiation did not reduce mortality (RR, 0.98; 95\% CI, 0.78-1.23), and secondary outcomes (ICU or hospital length of stay, renal function recovery, and RRT dependence) were not affected [51,52]. Even when five studies specific to CRRT were analyzed separately, early CRRT initiation did not significantly affect the outcome. In the analysis of other outcomes, except for overall mortality, the recommendation grade was weak due to poor quality and importance.

Similarly, another meta-analysis of nine RCTs comprising a total of 1,627 patients reported that earlier initiation of RRT had no benefit regarding mortality [53]. In subgroup analyses, in-hospital mortality decreased following early RRT initiation in surgical patients (RR, 0.78; 95\% CI, 0.640.95) and CRRT patients (RR, 0.80; 95\% CI, 0.66-0.95). Nevertheless, early intervention might not significantly improve the outcome but can increase the risk of side effects.

A study that analyzed a total of 1,479 patients from five RCTs, one prospective cohort study, and nine retrospective cohort studies published from 1971 to 2016, found that early RRT reduced 28-day mortality, ICU, and hospital lengths of stay, and RRT duration [54]. This was especially true if RRT was initiated within 12 hours or 24 hours in patients who developed AKI after cardiac surgery. Even after analyzing eight studies targeting only CRRT, the prognostic improvement effect of early RRT was confirmed (RR, 0.36; 95\% CI, 0.19-0.67). However, there are limitations to generalization because many retrospective studies were included, the number of patients was small, and different criteria were applied to each study.

According to the results of a review study comprising five RCTs and 1,084 participants published in the Cochrane Database of Systematic Reviews [55], early RRT seems to reduce the risk of death and improve renal function recovery. However, considering the $95 \%$ CI, early RRT can worsen the outcome, and an increase in adverse events due to early RRT was noted. In addition, the RR for death was 0.65 (95\% CI, 0.31-1.36) in the three RCTs for CRRT only, which was not statistically significant. The same was true for the recov- 
ery of renal function (RR, 1.36; 95\% CI, 0.79-2.34). Overall, most studies presented low-quality evidence, underscoring the need for adequately powered RCTs.

In a meta-analysis of nine studies published between April 2008 and December 2019, comprising 1,879 subjects [56], the timing of RRT initiation in the absence of an urgent indication did not affect the survival of critically ill patients with severe AKI (28-day mortality; HR, 1.01; 95\% CI, 0.87-1.17). The results were consistent regardless of sex, age, Sequential Organ Failure Assessment score, sepsis, or chronic kidney disease. Other outcomes and adverse events, such as death at 60 and 90 days, in-hospital death, duration of hospitalization, RRT-free days, RRT dependence upon discharge, and ventilator- and vasopressor-free days, were not affected by the timing of CRRT initiation.

A meta-analysis of 10 RCTs published between 2002 and 2020, including the most recently reported STARRT-AKI trial and comprising 4,753 patients, showed no correlation between the timing of RRT initiation and all-cause mortality or freedom from dialysis [57]. In the subgroup analyses, if the patient was in the surgical ICU or underwent CRRT, early initiation showed a benefit for the aforementioned outcomes. However, the studies used in this meta-analysis were heterogeneous, and there might have been biased; thus, the results should be interpreted with caution.

\section{Recommendations for future research}

To overcome the limitations of the previous studies and enable generalization of research results to clinical practice, we propose the following recommendations. First, the study population must be homogeneous; for instance, patients can be enrolled exclusively from medical or surgical ICUs or be limited to those receiving CRRT. The studies discussed in this paper included a mixture of patients receiving intermittent hemodialysis or CRRT, with few targeting CRRT only, hindering separate assessment of the findings for the two types of patients. Therefore, future RCTs should enroll more unified and homogenized study groups. Second, it is necessary to apply the inclusion and intervention (early or delayed) criteria realistically and conduct the research in a typical clinical situation where applicable. In other words, all patients with emergent indications should be included in the study. Third, multicenter and multinational studies should be conducted using well-designed protocols with minimal dependence upon subjective criteria and the judgments of clinicians and physicians when assigning treatments. Fourth, an RCT targeting older adults requiring CRRT is needed, considering the rapid aging of populations in many countries.

\section{Conclusions}

CRRT is important in the treatment and management of AKI in critically ill patients. Except for implementation in patients with life-threatening emergent indications, the proper timing of CRRT initiation remains controversial. In the current situation where it is difficult to generalize the conflicting results of recent research to patients seen in typical clinical practice and in the absence of reliable tools to

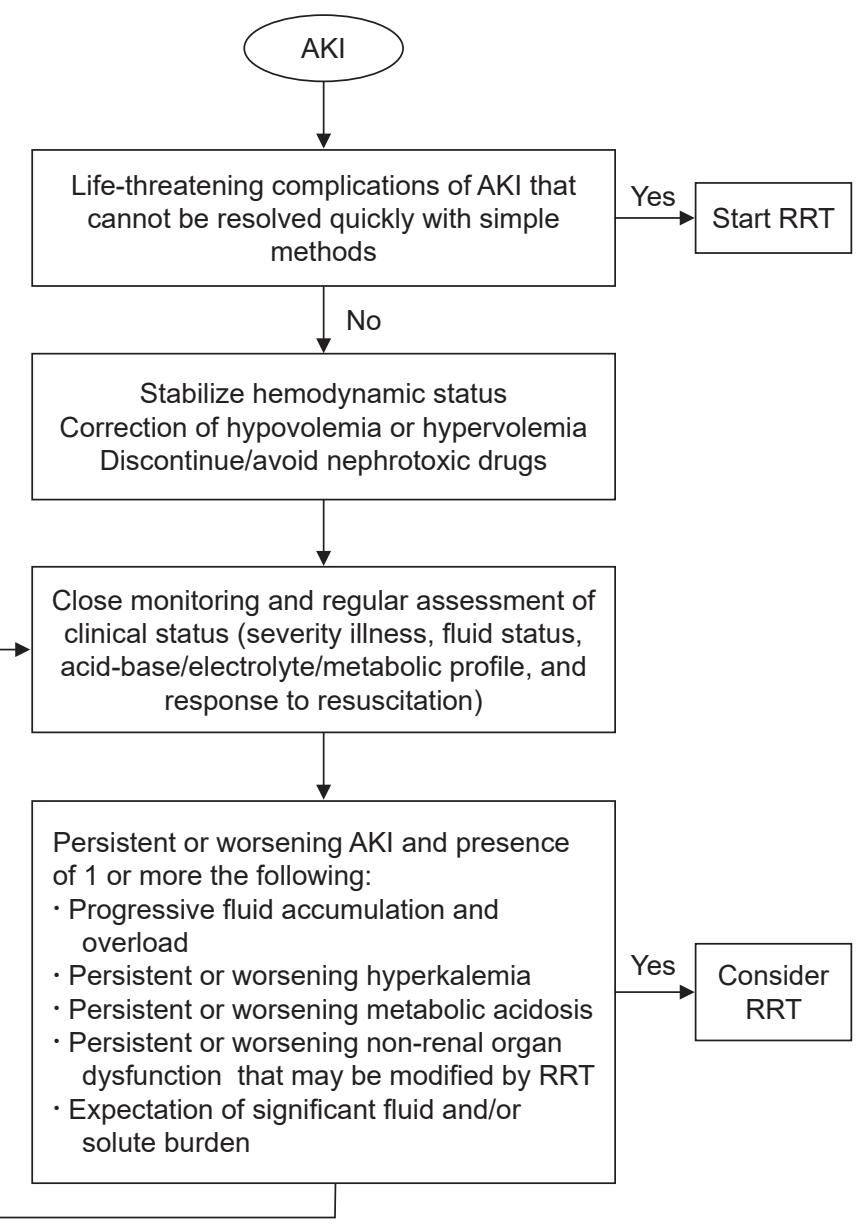

Figure 2. Clinical decision tree for initiation of RRT in critically ill patients with AKI. Reproduced from the article of Ostermann et al. (Contrib Nephrol 2016;187:106-120) [22] with the permission from S. Karger AG.

AKI, acute kidney injury; RRT, renal replacement therapy. 
predict the prognosis associated with RRT, a personalized patient-oriented approach should be used. In other words, it is important to determine the optimal timing of CRRT initiation by assessing the clinical situation and disease progression and not relying solely upon simple indicators such as serum creatinine concentration or urine volume.

The 17th Acute Disease Quality Initiative Consensus Group stated that acute RRT must be considered when metabolic and fluid demands exceed total kidney capacity and presented a conceptual model of demand-capacity balance [58]. The demand for kidney function is determined by nonrenal comorbidities, the severity of the acute disease, and the solute and fluid burden; the combination of these factors in given patients creates distinct scenarios requiring variable management. Demand-capacity imbalance is dynamic, varies from patient to patient, and should be evaluated regularly. In addition, selection of the preferred RRT modality is determined by the technological capability/availability at the healthcare facility, the inherent risk of the procedure, and current needs of the patient. In the case of CRRT, improved hemodynamic and intracranial pressure stability are expected benefits, but there are risks of infection, and patient immobilization is required. Notably, if the demand-capacity balance or treatment priorities change and an alternative technique are judged to be a better fit, transition to a different modality should be considered. All these processes underscore the need for a "precision \& personalized" approach, and this concept should be applied in real-world clinical practice in the future.

Recent research suggests that a careful waiting strategy should be properly applied according to patient (Fig. 2) [22], but CRRT should not be delayed if there is a life-threatening emergent condition. However, a wait-and-see approach with supportive care can be appropriate as there is no clear evidence that early CRRT initiation improves outcome, and CRRT is not a harmless treatment [59]. Therefore, future research related to the timing of CRRT initiation should focus on the development of algorithms to help clinicians make appropriate decisions that go beyond early or late CRRT initiation. It is expected that many ongoing studies will provide real-world data to support such algorithms and improve outcomes of critically ill patients with AKI in clinical practice.

\section{Conflicts of interest}

All authors have no conflicts of interest to declare.

\section{Authors' contributions}

Conceptualization: JNA, YRS

Data curation, Formal analysis: All authors

Writing-original draft: JNA

Writing-review \& editing: All authors

All authors read and approved the final manuscript.

\section{ORCID}

Jung Nam An, https://orcid.org/0000-0001-5108-1005

Sung Gyun Kim, https://orcid.org/0000-0002-5034-0527

Young Rim Song, https://orcid.org/0000-0002-0416-4745

\section{References}

1. Siew ED, Davenport A. The growth of acute kidney injury: a rising tide or just closer attention to detail? Kidney Int 2015;87:4661.

2. Tolwani A. Continuous renal-replacement therapy for acute kidney injury. N Engl J Med 2012;367:2505-2514.

3. Chawla LS, Amdur RL, Amodeo S, Kimmel PL, Palant CE. The severity of acute kidney injury predicts progression to chronic kidney disease. Kidney Int 2011;79:1361-1369.

4. An JN, Hwang JH, Kim DK, et al. Chronic kidney disease after acute kidney injury requiring continuous renal replacement therapy and its impact on long-term outcomes: a multicenter retrospective cohort study in Korea. Crit Care Med 2017;45:47-57.

5. Chawla LS, Amdur RL, Shaw AD, Faselis C, Palant CE, Kimmel PL. Association between AKI and long-term renal and cardiovascular outcomes in United States veterans. Clin J Am Soc Nephrol 2014;9:448-456.

6. Wu VC, Wu CH, Huang TM, et al. Long-term risk of coronary events after AKI. J Am Soc Nephrol 2014;25:595-605.

7. Wu VC, Wu PC, Wu CH, et al. The impact of acute kidney injury on the long-term risk of stroke. J Am Heart Assoc 2014;3:e000933.

8. Lai TS, Wang CY, Pan SC, et al. Risk of developing severe sepsis after acute kidney injury: a population-based cohort study. Crit Care 2013;17:R231.

9. Mehta RL, Bouchard J, Soroko SB, et al. Sepsis as a cause and consequence of acute kidney injury: program to improve care in 
acute renal disease. Intensive Care Med 2011;37:241-248.

10. Wu PC, Wu CJ, Lin CJ, Wu VC; National Taiwan University Study Group on Acute Renal Failure Group. Long-term risk of upper gastrointestinal hemorrhage after advanced AKI. Clin J Am Soc Nephrol 2015;10:353-362.

11. Park S, Lee S, Jo HA, et al. Epidemiology of continuous renal replacement therapy in Korea: results from the National Health Insurance Service claims database from 2005 to 2016. Kidney Res Clin Pract 2018;37:119-129.

12. Wald R, McArthur E, Adhikari NK, et al. Changing incidence and outcomes following dialysis-requiring acute kidney injury among critically ill adults: a population-based cohort study. Am J Kidney Dis 2015;65:870-877.

13. Hsu RK, McCulloch CE, Dudley RA, Lo LJ, Hsu CY. Temporal changes in incidence of dialysis-requiring AKI. J Am Soc Nephrol 2013;24:37-42.

14. Kolhe NV, Muirhead AW, Wilkes SR, Fluck RJ, Taal MW. National trends in acute kidney injury requiring dialysis in England between 1998 and 2013. Kidney Int 2015;88:1161-1169.

15. Bagshaw SM, Laupland KB, Doig CJ, et al. Prognosis for longterm survival and renal recovery in critically ill patients with severe acute renal failure: a population-based study. Crit Care 2005;9:R700-R709.

16. Uchino S, Kellum JA, Bellomo R, et al. Acute renal failure in critically ill patients: a multinational, multicenter study. JAMA 2005;294:813-818.

17. Liu KD, Himmelfarb J, Paganini E, et al. Timing of initiation of dialysis in critically ill patients with acute kidney injury. Clin J Am Soc Nephrol 2006;1:915-919.

18. Saudan P, Niederberger M, De Seigneux S, et al. Adding a dialysis dose to continuous hemofiltration increases survival in patients with acute renal failure. Kidney Int 2006;70:1312-1317.

19. Hyman A, Mendelssohn DC. Current Canadian approaches to dialysis for acute renal failure in the ICU. Am J Nephrol 2002;22:2934.

20. Lins RL, Elseviers MM, Van der Niepen P, et al. Intermittent versus continuous renal replacement therapy for acute kidney injury patients admitted to the intensive care unit: results of a randomized clinical trial. Nephrol Dial Transplant 2009;24:512-518.

21. Jhee JH, Park JY, An JN, et al. Cumulative fluid balance and mortality in elderly patients with acute kidney injury requiring continuous renal-replacement therapy: a multicenter prospective cohort study. Kidney Res Clin Pract 2020;39:414-425.

22. Ostermann M, Wald R, Bagshaw SM. Timing of renal replacement therapy in acute kidney injury. Contrib Nephrol
2016;187:106-120.

23. Clark E, Wald R, Walsh M, Bagshaw SM; Canadian Acute Kidney Injury (CANAKI) Investigators. Timing of initiation of renal replacement therapy for acute kidney injury: a survey of nephrologists and intensivists in Canada. Nephrol Dial Transplant 2012;27:2761-2767.

24. Wald R, Adhikari NK, Smith OM, et al. Comparison of standard and accelerated initiation of renal replacement therapy in acute kidney injury. Kidney Int 2015;88:897-904.

25. Prowle JR, Davenport A. Does early-start renal replacement therapy improve outcomes for patients with acute kidney injury? Kidney Int 2015;88:670-673.

26. Gettings LG, Reynolds HN, Scalea T. Outcome in post-traumatic acute renal failure when continuous renal replacement therapy is applied early vs. late. Intensive Care Med 1999;25:805-813.

27. Durmaz I, Yagdi T, Calkavur T, et al. Prophylactic dialysis in patients with renal dysfunction undergoing on-pump coronary artery bypass surgery. Ann Thorac Surg 2003;75:859-864.

28. Sugahara S, Suzuki H. Early start on continuous hemodialysis therapy improves survival rate in patients with acute renal failure following coronary bypass surgery. Hemodial Int 2004;8:320-325.

29. Elahi MM, Lim MY, Joseph RN, Dhannapuneni RR, Spyt TJ. Early hemofiltration improves survival in post-cardiotomy patients with acute renal failure. Eur J Cardiothorac Surg 2004;26:10271031.

30. Demirkiliç U, Kuralay E, Yenicesu M, et al. Timing of replacement therapy for acute renal failure after cardiac surgery. J Card Surg 2004;19:17-20.

31. Manché A, Casha A, Rychter J, Farrugia E, Debono M. Early dialysis in acute kidney injury after cardiac surgery. Interact Cardiovasc Thorac Surg 2008;7:829-832.

32. Wang X, Jie Yuan W. Timing of initiation of renal replacement therapy in acute kidney injury: a systematic review and meta-analysis. Ren Fail 2012;34:396-402.

33. Vaara ST, Reinikainen M, Wald R, Bagshaw SM, Pettilä V; FINNAKI Study Group. Timing of RRT based on the presence of conventional indications. Clin J Am Soc Nephrol 2014;9:1577-1585.

34. Libório AB, Leite TT, Neves FM, Teles F, Bezerra CT. AKI complications in critically ill patients: association with mortality rates and RRT. Clin J Am Soc Nephrol 2015;10:21-28.

35. Yoon BR, Leem AY, Park MS, Kim YS, Chung KS. Optimal timing of initiating continuous renal replacement therapy in septic shock patients with acute kidney injury. Sci Rep 2019;9:11981.

36. Karakala N, Tolwani AJ. Timing of renal replacement therapy for 
acute kidney injury. J Intensive Care Med 2019;34:94-103.

37. Bagshaw SM, Uchino S, Bellomo R, et al. Timing of renal replacement therapy and clinical outcomes in critically ill patients with severe acute kidney injury. J Crit Care 2009;24:129-140.

38. Vats HS, Dart RA, Okon TR, Liang H, Paganini EP. Does early initiation of continuous renal replacement therapy affect outcome: experience in a tertiary care center. Ren Fail 2011;33:698-706.

39. Clec'h C, Darmon M, Lautrette A, et al. Efficacy of renal replacement therapy in critically ill patients: a propensity analysis. Crit Care 2012;16:R236.

40. Wilson FP, Yang W, Machado CA, et al. Dialysis versus nondialysis in patients with AKI: a propensity-matched cohort study. Clin J Am Soc Nephrol 2014;9:673-681.

41. Wang C, Lv LS, Huang H, et al. Initiation time of renal replacement therapy on patients with acute kidney injury: a systematic review and meta-analysis of 8179 participants. Nephrology (Carlton) 2017;22:7-18.

42. Section 5: dialysis interventions for treatment of AKI. Kidney Int Suppl (2011) 2012;2:89-115.

43. Vinsonneau C, Allain-Launay E, Blayau C, et al. Renal replacement therapy in adult and pediatric intensive care: recommendations by an expert panel from the French Intensive Care Society (SRLF) with the French Society of Anesthesia Intensive Care (SFAR) French Group for Pediatric Intensive Care Emergencies (GFRUP) the French Dialysis Society (SFD). Ann Intensive Care 2015;5:58.

44. Doi K, Nishida O, Shigematsu T, et al. The Japanese Clinical Practice Guideline for acute kidney injury 2016. J Intensive Care 2018;6:48.

45. Guideline Updates Team (UK). Acute kidney injury: prevention, detection and management. London: National Institute for Health and Care Excellence (UK); 2019.

46. Zarbock A, Kellum JA, Schmidt C, et al. Effect of early vs delayed initiation of renal replacement therapy on mortality in critically ill patients with acute kidney injury: the ELAIN randomized clinical trial. JAMA 2016;315:2190-2199.

47. Gaudry S, Hajage D, Schortgen F, et al. Initiation strategies for renal-replacement therapy in the intensive care unit. $N$ Engl J Med 2016;375:122-133.

48. Barbar SD, Clere-Jehl R, Bourredjem A, et al. Timing of renal-replacement therapy in patients with acute kidney injury and sepsis. N Engl J Med 2018;379:1431-1442.
49. STARRT-AKI Investigators; Canadian Critical Care Trials Group;; Australian and New Zealand Intensive Care Society Clinical Trials Group, et al. Timing of initiation of renal-replacement therapy in acute kidney injury. N Engl J Med 2020;383:240-251.

50. Gaudry S, Hajage D, Martin-Lefevre L, et al. The Artificial Kidney Initiation in Kidney Injury 2 (AKIKI2): study protocol for a randomized controlled trial. Trials 2019;20:726.

51. Yang XM, Tu GW, Zheng JL, et al. A comparison of early versus late initiation of renal replacement therapy for acute kidney injury in critically ill patients: an updated systematic review and meta-analysis of randomized controlled trials. BMC Nephrol 2017;18:264.

52. Feng YM, Yang Y, Han XL, Zhang F, Wan D, Guo R. The effect of early versus late initiation of renal replacement therapy in patients with acute kidney injury: a meta-analysis with trial sequential analysis of randomized controlled trials. PLoS One 2017;12:e0174158.

53. Lai TS, Shiao CC, Wang JJ, et al. Earlier versus later initiation of renal replacement therapy among critically ill patients with acute kidney injury: a systematic review and meta-analysis of randomized controlled trials. Ann Intensive Care 2017;7:38.

54. Zou H, Hong Q, Xu G. Early versus late initiation of renal replacement therapy impacts mortality in patients with acute kidney injury post cardiac surgery: a meta-analysis. Crit Care 2017;21:150.

55. Fayad AII, Buamscha DG, Ciapponi A. Timing of renal replacement therapy initiation for acute kidney injury. Cochrane Database Syst Rev 2018;12:CD010612.

56. Gaudry S, Hajage D, Benichou N, et al. Delayed versus early initiation of renal replacement therapy for severe acute kidney injury: a systematic review and individual patient data meta-analysis of randomised clinical trials. Lancet 2020;395:1506-1515.

57. Pan HC, Chen YY, Tsai IJ, et al. Accelerated versus standard initiation of renal replacement therapy for critically ill patients with acute kidney injury: a systematic review and meta-analysis of RCT studies. Crit Care 2021;25:5.

58. Ostermann M, Joannidis M, Pani A, et al. Patient selection and timing of continuous renal replacement therapy. Blood Purif 2016;42:224-237.

59. Vanmassenhove J, Vanholder R, Van Biesen W, Lameire N. Haste makes waste: should current guideline recommendations for initiation of renal replacement therapy for acute kidney injury be changed? Semin Dial 2018;31:204-208. 